JOURNAL OF

SYMPLECTIC GEOMETRY

Volume 8, Number 1, 57-65, 2010

\title{
NOTE ON GEODESIC RAYS AND SIMPLE TEST CONFIGURATIONS
}

\author{
SONG SUN
}

In this short note, we give a new proof of a theorem of Arezzo-Tian on the existence of smooth geodesic rays tamed by a special degeneration with smooth central fiber.

In [7], Donaldson proposed a program to tackle the problem of the existence and uniqueness of extremal metrics on a Kähler manifold from the perspective of the infinite dimensional space of Kähler potentials. He observed that the existence of smooth geodesics connecting two arbitrary Kähler potentials implies the uniqueness of Kähler metrics in the given class with constant scalar curvature. In [3], Chen proved the existence of $C^{1,1}$ geodesics joining two arbitrary points in $\mathcal{H}$. Consequently, this established the uniqueness of extremal Kähler metrics when the first Chern class of the manifold is non-positive. At present, there is extensive research in this direction. In particular, the uniqueness problem has been completely settled (cf. $[\mathbf{6}, \mathbf{1 0}, \mathbf{1 2}])$.

We shall first give a very brief outline about a small part of this program, which is directly relevant to the problem at hand. For more detailed accounts, readers are referred to $[\mathbf{3}, \mathbf{4}, \mathbf{6}, \mathbf{7}]$.

Let $(M, \omega, J)$ be an $n$-dimensional Kähler manifold. Define the infinite dimensional space of Kähler potentials as

$$
\mathcal{H}=\left\{\phi \in C^{\infty}(M) \mid \omega_{\phi}=\omega+\sqrt{-1} \partial \bar{\partial} \phi>0\right\} .
$$

In [11] (cf. [7, 16]), Mabuchi first introduced a Weil-Petersson type metric on $\mathcal{H}$ :

$$
\left(\phi_{1}, \phi_{2}\right)_{\phi}=\int_{M} \phi_{1} \phi_{2} \frac{\omega_{\phi}^{n}}{n !}
$$


where $\phi_{1}, \phi_{2} \in T_{\phi} \mathcal{H} \simeq C^{\infty}(M)$. It is easy to see that the geodesic equation in $\mathcal{H}$ is

$$
\ddot{\phi}=\frac{1}{2}\left|\nabla_{\phi} \dot{\phi}\right|_{\phi}^{2}
$$

A straightforward calculation shows (cf. $[\mathbf{7}, \mathbf{1 1}, \mathbf{1 6}])$ that the space $\mathcal{H}$ is formally of non-positive curvature. This fact was made rigorously in $[\mathbf{2}]$, where E. Calabi and X-X. Chen proved that $\mathcal{H}$ is a non-positively curved space in the sense of Alexanderov.

According to Semmes [16], by adding a trivial $S^{1}$ factor, the geodesic equation could be written as a degenerate complex Monge-Ampère equation in $M \times\left([0,1] \times S^{1}\right)$. Suppose $X$ is a Riemann surface with boundary. Denote $\pi_{1}: M \times X \rightarrow M$ and $\pi_{2}: M \times X \rightarrow X$ as the two natural projection maps, and let $\Omega=\pi_{1}^{*} \omega$. Then, given $\phi_{0} \in C^{\infty}(M \times \partial X)$ such that $\Omega+\sqrt{-1} \partial \bar{\partial} \phi_{0}>$ 0 on each slice $M \times\{x\}$ for all $x \in \partial X$, we consider the Dirichlet boundary value problem:

$$
\begin{cases}(\Omega+\sqrt{-1} \partial \bar{\partial} \Phi)^{n+1}=0, & \text { on } M \times X, \\ \Phi=\phi_{0}, & \text { on } M \times \partial X .\end{cases}
$$

A solution is of geometric interest if $\Omega+\sqrt{-1} \partial \bar{\partial} \Phi>0$ when restricted on each slice $M \times\{x\}$ for all $x \in X$. Since the target manifold $\mathcal{H}$ is an infinitesimal symmetric space, any smooth solution of (2) can be re-interpreted (cf. [7]) as a harmonic map from $X$ to $\mathcal{H}$ with prescribed boundary map $\phi_{0}: \partial X \rightarrow \mathcal{H}$. Any geodesic segment connecting $\phi_{1}$ with $\phi_{2}$ corresponds to an $S^{1}$ invariant solution of (2) with $X=[0,1] \times S^{1}$ and $\phi_{0}(0, \tau)=\phi_{1}(\tau)$, $\phi_{0}(1, \tau)=\phi_{2}(\tau)$. The notion of a geodesic ray is similar to the finite dimensional case: a geodesic ray in $\mathcal{H}$ is a geodesic segment which can be infinitely extended in one direction. In other words, a geodesic ray corresponds to an $S^{1}$ invariant solution of the following:

$$
(\Omega+\sqrt{-1} \partial \bar{\partial} \Phi)^{n+1}=0, \text { on } M \times\left([0, \infty) \times S^{1}\right) \simeq M \times(D \backslash\{0\}),
$$

where $D$ is the closed unit disk.

In [7], Donaldson also conjectured that the existence of smooth geodesic rays where the $\mathrm{K}$ energy is strictly decreasing at the infinity is equivalent to the non-existence of constant scalar curvature metrics in $[\omega]$. Donaldson's conjecture certainly motivated the study of the existence of geodesic rays and related problems. However, the existence of geodesic rays is quite different from that of geodesic segments since the domain involved is naturally non-compact. More importantly, Donaldson [7] pointed out that the initial value problem for the geodesic ray equation is not always solvable in the smooth category. So we need to impose an alternative condition in order to properly solve equation (3). Following Donaldson's program [7], this issue was discussed in [4]. According to [4], the initial Kähler potential together with the asymptotic direction (given by either an existing geodesic ray or an 
algebraic ray associated to a test configuration) forms a well-posed Dirichlet boundary value for equation (3). A set of new problems were discussed there for developing the existence theory of geodesic rays. In particular, it is proved that the existence of relative $C^{1,1}$ geodesic rays parallel to a given smooth geodesic ray under natural geometrical constraints. Unfortunately, there are few examples of the existence of geodesic rays in the literature, which creates serious problem for pushing the general existence theory further. In $[\mathbf{1}]$, using Cauchy-Kowalewski theorem, Arezzo and Tian proved the existence of a smooth geodesic ray asymptotically parallel to a special degeneration with smooth central fiber, or equivalently, to a simple test configuration (cf. $[\mathbf{5}, \mathbf{9}])$. One would like to see a more direct PDE proof of this important theorem. The main purpose of this note is to reprove the same theorem using perturbation argument. Note that if we only assume the total space of the test configuration to be smooth, then by [5], it will give rise to a relative $C^{1,1}$ geodesic ray parallel to the algebraic ray induced by the degeneration. Furthermore, it was shown in [5] that even for tori varieties, the best regularity one could get is $C^{1,1}$ (see also [17]). There are also other ways of obtaining geodesic rays from a test configuration, see $[\mathbf{1 4}, \mathbf{1 5}]$.

Now we introduce the definition of a Kähler fibration and a simple test configuration.

Definition 1. A Kähler fibration (over the closed unit disk) is a map $\pi$ : $(\mathcal{M}, J, \Omega) \rightarrow D$, where $J$ is an integrable complex structure on $\mathcal{M}, \pi$ is a holomorphic submersion, $\Omega$ is a closed two form on $\mathcal{M}$ which is compatible with $J$ and it is a Kähler form on each fiber $M_{z}(z \in D)$ (which is assumed to be compact without boundary).

Definition 2 (cf. $[\mathbf{5}, \mathbf{9}]$ ). A (truncated) simple test configuration for a polarized Kähler manifold $L \rightarrow M$ is a Kähler fibration $\pi:(\mathcal{M}, J, \Omega) \rightarrow D$ together with a very ample line bundle $\mathcal{L}$ and a $\mathbb{C}^{*}$ equivariant embedding $\{\mathcal{L} \rightarrow \mathcal{M} \rightarrow D\} \hookrightarrow\left\{\mathcal{O}(1) \rightarrow \mathbb{P}^{N} \times \mathbb{C} \rightarrow \mathbb{C}\right\}$, such that $\{L \rightarrow M\}$ is isomorphic $\left\{\left.\mathcal{L}\right|_{M_{1}} \rightarrow M_{1}\right\}$, where we denote $M_{t}=\pi^{-1}(t)$. Also, the $\mathbb{C}^{*}$ action on $\mathbb{C}$ is given by the standard multiplication, and the map $\mathbb{P}^{N} \times \mathbb{C} \rightarrow \mathbb{C}$ is simply the projection to the second factor. In addition, $\Omega$ should coincide with the restriction of the Fubini-Study metric on $\mathbb{P}^{N}$, while the induced $S^{1}$ actions on all these spaces are assumed to be unitary. Clearly all the fibers $\pi^{-1}(t)$ for $t \neq 0$ are biholomorphic to each other. A simple test configuration is called product if $\mathcal{M}$ is biholomorphic to $M \times \mathbb{C}$, and the $\mathbb{C}^{*}$ action on $\mathcal{M}$ is also a product action coming from $\mathbb{C}^{*}$ action on $M$ and the standard multiplication on $\mathbb{C}$. It is called trivial if the $\mathbb{C}^{*}$ action on $\mathcal{M}$ is also trivial.

Remark 3. The above definition of a simple test configuration is essentially the same as the special degeneration with smooth central fiber studied by Tian first in [18]. 
Theorem 4 (Arezzo-Tian [1]). Given a non-trivial simple test configuration for $L \rightarrow M$, there exists a non-trivial geodesic ray tamed by this test configuration.

According to [4], a geodesic ray is said to be tamed by a test configuration if it is asymptotically parallel to the algebraic ray defined by pulling back the Kähler potentials through the $\mathbb{C}^{*}$ action on $\mathcal{M}$.

We want to take a different route to prove this theorem. Following $[\mathbf{5}, \mathbf{8}]$, smooth regular solutions to (3) are related to foliations of punctured holomorphic discs with some control on the total area. There is a Fredholm theory associated to the moduli space of holomorphic discs with totally real boundary condition. Deformation of this moduli space is the central topic of this note.

Arezzo-Tian's theorem is a consequence of the following proposition.

Proposition 5. let $\pi:(\mathcal{M}, J, \Omega) \rightarrow D$ be a Kähler fibration, there exists a smooth function $\Phi$ defined in a neighborhood of the central fiber $M_{0}$ that solves the complex Monge-Ampère equation $(\Omega+\sqrt{-1} \partial \bar{\partial} \Phi)^{n+1}=0$ with $\Omega+\sqrt{-1} \partial \bar{\partial} \Phi$ being positive on each fiber.

In [1], it was shown that the value of $\Phi$ on the central fiber could be prescribed as long as the corresponding Kähler metric is real analytic. From the proof of Proposition 5 we shall see that also more is true. We only state it in the following $S^{1}$ invariant case, since this gives rise to geodesic rays. Namely,

Theorem 6. Let $\pi:(\mathcal{M}, J, \Omega) \rightarrow D$ be a non-trivial simple test configuration. Denote $M_{z}=\pi^{-1}(z)$ for $z \in D$, and $\omega_{z}=\left.\Omega\right|_{M_{z}}$. For $k>0$ sufficiently large, denote $\tilde{\mathcal{H}}_{0}=\left\{\phi \in C^{k+1}\left(M_{0} ; \mathbb{R}\right) \mid \phi\right.$ is $S^{1}$ invariant and $\left.\omega_{0}+\sqrt{-1} \partial \bar{\partial} \phi>0\right\}$. Given any $\phi_{0} \in \tilde{\mathcal{H}}_{0}$, there is an open set $\mathcal{U}$ in $\tilde{\mathcal{H}}_{0}$ and a number $\delta(k)>0$, such that for every $\phi \in \mathcal{U}$ there exists a $S^{1}$ invariant $\Phi \in C^{k}\left(\pi^{-1}(|z| \leq \delta)\right)$ depending on $\phi$ that solves the complex Monge-Ampère equation $(\Omega+\sqrt{-1} \partial \bar{\partial} \Phi)^{n+1}=0$ with $\Omega+\sqrt{-1} \partial \bar{\partial} \Phi$ being positive on each fiber.

The proof of Proposition 5 is based on a perturbation theory first introduced in [8] by Donaldson in the case of a trivial test configuration. In this note, we follow its generalization in [5]. By the definition of a Kähler fibration, $\mathcal{M}$ is always diffeomorphic to the product $M_{0} \times D$. So we can for simplicity assume $\mathcal{M}=M \times D$ for a $2 n$ dimensional smooth manifold $M$ and the map $\pi$ involved in the definition is the projection map to the second factor. Fix once and for all a cover of $M \times D$ by small balls, say $\left\{U_{i}\right\}_{i \in I}$. Following Donaldson's construction, we can associate a manifold $\mathcal{W}$ to any Kähler fibration, as follows: On each $U_{i}$, we choose local holomorphic coordinates to be $\left(z_{1}, \ldots, z_{n}, z\right)$, where $z$ is simply given by $\pi$. Then $\Omega$ could be written as $\sqrt{-1} \partial \bar{\partial} \rho_{i}$ for some locally defined function $\rho_{i}$. $\mathcal{W}$ is obtained by 
twisting the vertical holomorphic cotangent bundle $E=T^{*}(M \times D) / \pi^{*} T^{*} D$. More precisely, we glue $\xi$ in $\left.E\right|_{U_{i}}$ with $\xi+\partial\left(\rho_{i}-\rho_{j}\right)$ in $\left.E\right|_{U_{j}}$ over the corresponding fiber. It is easy to see that $\mathcal{W}$ is also a fibration over $D$ and the canonical complex-symplectic structure on the holomorphic cotangent bundle induces a fiberwise complex-symplectic form on $\mathcal{W}$. Furthermore, $\Omega$ defines an exact LS-graph ${ }^{1}$ on each vertical fiber.

Of course, our construction of $\mathcal{W}$ is not canonical. However, if we fix an open cover and an initial Kähler fibration, then $\rho_{i}$ could be chosen to depend smoothly on the data $\Omega$ and $J$ for a small variation. (Indeed, by the wellknown theorem of Newlander-Nirenberg, holomorphic coordinates could be made to vary smoothly. Then, one can follow the proof of Dolbeault's lemma to show this.) Moreover, by definition, $\mathcal{W}$ is always diffeomorphic to $E$, or further, to the real vertical cotangent bundle, still denoted by $E$, which is independent of $\Omega$ and $J$. Therefore, if we pull back everything to the latter, a perturbation of $\Omega$ and $J$ really gives us a perturbation of the complexsymplectic structure on $E$.

Now let $\phi_{0}: \partial D \rightarrow \mathbb{R}$ be a smooth function such that $\Omega+\sqrt{-1} \partial \bar{\partial} \phi_{0}$ is positive on fibers over $\partial D$. Then it defines exact LS-graphs $\Lambda_{z, \phi_{0}}$ over any $z \in \partial D$. Following $[\mathbf{5}, \mathbf{8}]$, we have a one-to-one correspondence:

(A) A $C^{\infty}$ solution $\Phi$ to the homogeneous Monge-Ampère equation: $(\Omega+$ $\sqrt{-1} \partial \bar{\partial} \Phi)^{n+1}=0$ satisfying the boundary condition $\left.\Phi\right|_{\partial D}=\phi_{0}$ and such that $\Omega+\sqrt{-1} \partial \bar{\partial} \Phi$ still defines a Kähler fibration (together with $J$ ).

(B) A smooth map $G: M \times D \rightarrow E$ which covers the identity map on $D$, holomorphic in the second variable and satisfies the boundary condition: for all $z \in \partial D, G(\cdot, z) \in \Lambda_{z, \phi_{0}}$. (Alternatively, we could view this as a family of holomorphic sections of the fibration $E \rightarrow D$ whose boundary lies in some totally real submanifold given by $\bigcup_{z \in \partial D} \Lambda_{z, \phi_{0}}$.) In addition, we require that $p_{1} \circ G(\cdot, 0)$ is the identity map, and $p_{1} \circ G(\cdot, z)$ is a diffeomorphism for any $z \in D$, where $p_{1}: E \rightarrow M$ is the projection map.

Lemma 7. Perturbation of $\Omega, J$ and $\phi_{0}$ preserves a smooth solution to the above equation, i.e., the compact family of normalized holomorphic discs in (B) is stable under perturbation.

To prove this lemma, we need to set up a Fredholm theory for holomorphic discs with totally real boundary conditions. Denote by $(D, E)_{s}$ the space of maps from $D$ to $E$ which lies in the Sobolev space $H^{s+1}$ for some large $s$. Let $\mathcal{F}_{s}$ be the subspace of $(D, E)_{s}$ which are sections of the fibration, i.e., normalized maps. Fix $J_{0}$ on $E$, and a totally real submanifold $R_{0}$ of $E$ with respect to $J_{0}$. (For example, in our case, the exact Lagrangian graphs defined by the known smooth solution restricted on $\partial D$.) Denote by $N R_{0}$

\footnotetext{
${ }^{1}$ In a complex symplectic manifold $(M, \Theta)$, a submanifold $L$ is called an $L S$-submanifold if $L$ is Lagrangian with respect to $\operatorname{Re} \Theta$, while the restriction of $\operatorname{Im} \Theta$ on $L$ is a symplectic form. For more details, see $[\mathbf{5}, \mathbf{8}]$.
} 
the normal bundle of $R_{0}$ in $E$ with respect to any fixed metric, then a $C^{\infty}$ neighborhood of totally real submanifolds around $R_{0}$ can be identified with an open set of the space $\Gamma\left(N R_{0}\right)$ of all smooth sections of $N R_{0}$. As in [13], choose $\bar{\epsilon}=\left\{\epsilon_{k}\right\}_{k \in \mathbb{N}}\left(\epsilon_{k} \rightarrow 0\right)$, and define a Floer norm on $\Gamma\left(N R_{0}\right)$ by

$$
\|X\|_{\bar{\epsilon}}=\sum_{k \in \mathbb{N}} \epsilon_{k} \max _{x \in E}\left|D^{k} X(x)\right| .
$$

For $r>0$, we also define

$$
\Gamma_{r}^{\bar{\epsilon}}\left(N R_{0}\right)=\left\{X \in \Gamma\left(N R_{0}\right) \mid\|X\|_{\bar{\epsilon}}<r\right\} .
$$

This is a Banach space and by choosing $r$ sufficiently small, we can assume that $\Gamma_{r}^{\bar{\epsilon}}\left(N R_{0}\right)$ maps injectively to the space of all totally real submanifolds of $E$, under the previous identification. Let $\mathcal{N}\left(R_{0}\right)$ denote its image. For each $R \in \mathcal{N}\left(R_{0}\right)$, there is an associated diffeomorphism $\phi_{R}: R \rightarrow R_{0}$ which extends to a diffeomorphism of $E$. Moreover, we can choose $\phi_{R}$ to depend smoothly on $R$. Now let $\mathcal{B}=\cup_{u \in \mathcal{F}_{s}} H^{s}\left(u^{*} T E\right)$ be an infinite dimensional vector bundle over $\mathcal{F}_{s}$, and $\mathcal{J}$ be the space of smooth almost complex structures on $E$, which are $C^{\bar{\epsilon}}$ close to $J_{0}$. Then $\mathcal{B} \times(\partial D, E)_{s-\frac{1}{2}}$ is a bundle over $\mathcal{F}_{s} \times \mathcal{J} \times \mathcal{N}\left(R_{0}\right)$, with a section $s(u, J, R)=\left(\bar{\partial}_{J} u,\left.\phi_{R}^{-1} \circ u\right|_{\partial D}\right)$. Fix $J_{0}$, and let $s_{0}$ be the restriction of $s$ to the slice $\mathcal{F}_{s} \times\left\{J_{0}\right\} \times \mathcal{N}\left(R_{0}\right)$. It is shown in [13] that $s_{0}$ is transversal to the submanifold $\{0\} \times\left(\partial D, R_{0}\right)_{s-\frac{1}{2}}$ at a point $\left(u_{0}, R_{0}\right)$ if $u_{0}$ is not multiply covered, i.e., there exists a $z \in \partial D$, such that $u_{0}^{-1}\left(u_{0}(z)\right) \cap \partial D=z$ and $D u_{0}(z) \neq 0$. So in our particular case $s$ is transversal to $\{0\} \times\left(\partial D, R_{0}\right)_{s-\frac{1}{2}}$ at $\left(u_{0}, J_{0}, R_{0}\right)$ for every disc coming from a solution of our previous equation $(\mathrm{A})$. Therefore, $s^{-1}\left(\{0\} \times\left(\partial D, R_{0}\right)_{s-\frac{1}{2}}\right)$ is smooth Banach manifold near $\left(u_{0}, J_{0}, R_{0}\right)$. Standard elliptic regularity implies that it is actually contained in $\mathcal{F}_{\infty} \times \mathcal{J} \times \mathcal{N}\left(R_{0}\right)$ for $s$ sufficiently big.

Now consider the projection map $s^{-1}\left(\{0\} \times\left(\partial D, R_{0}\right)_{s-\frac{1}{2}}\right) \rightarrow \mathcal{J} \times \mathcal{N}\left(R_{0}\right)$, which is Fredholm of index $2 n$ (cf. $[\mathbf{5}, \mathbf{8}])$. Given a smooth solution on $(M \times$ $D, J, \Omega)$ as in (A), we have a $2 n$ dimensional compact family of normalized holomorphic discs into $\left(E, J_{0}\right)$, where $J_{0}$ is defined by $J$ and $\Omega$. Moreover, the holomorphic discs appearing in the family are all super-regular, ${ }^{2}$ and in particular regular. Now if we perturb $J, \Omega$ and $\phi_{0}$, we are actually perturbing $J_{0}$ and $R_{0}$. Standard Fredholm theory ensures the existence of a nearby family of normalized regular holomorphic discs, which proves Lemma 7.

Proof of Proposition 5. For $r \in(0,1)$, let $\mathcal{M}(r)$ be the re-scaled Kähler fibration defined by $\left.(\mathcal{M}, J, \Omega)\right|_{|z| \leq r}$ with $\pi_{r}(w)=\pi(w) / r$. When $r$ is

\footnotetext{
${ }^{2}$ For a family of holomorphic $\operatorname{discs} G: M \times D \rightarrow \mathcal{W}$ parameterized by $M$, we say that a disc $G_{x}(x \in M)$ is super-regular if the derivative $d p_{1} \circ d_{x} G(\cdot, z): T_{x} M \rightarrow T_{p_{1} \circ G(x, z)} M$ is surjective for all $z \in D$. It is proved in $[\mathbf{5}, \mathbf{8}]$, that a super-regular disc is automatically regular.
} 
small enough, $\mathcal{M}(r)$ is close to the trivial fibration given by the product $\left(M_{0},\left.J\right|_{M_{0}},\left.\Omega\right|_{M_{0}}\right) \times D$. The latter has an obvious solution to $(A)$ (just take $\Phi=0)$. Therefore by Lemma 7 , for $r$ small, we obtain a solution to the equation on $\mathcal{M}(r)$, which is the same as a solution near the central fiber on $\mathcal{M}$.

Proof of Theorem 4. The limit of the re-scaled test configurations $\mathcal{M}(r)$ is $\left(M_{0},\left.J\right|_{M_{0}},\left.\Omega\right|_{M_{0}}\right) \times D$ with a (possibly non-trivial) $C^{*}$ action on $M_{0}$. Any $S^{1}$ invariant potential $\phi_{0}$ on $M_{0}$ yields a trivial solution. We can perturb $\phi_{0}$ to an $S^{1}$ invariant function $\phi$ on $\partial \mathcal{M}(r)$ for small $r$, then the solution $\Phi$ ensured by Proposition 5 will also be $S^{1}$ invariant by the uniqueness of solutions of equation (2), which follows from a standard maximum principle argument (see Lemma 6 in [7]). Then we obtain a geodesic ray on the fiber $M_{1}$ by pulling back the restriction of $\Omega+\sqrt{-1} \partial \bar{\partial} \Phi$ on each fiber to a fixed fiber by the $\mathbb{C}^{*}$ action, and we also get a foliation by punctured holomorphic discs on $M_{1} \times(D \backslash\{0\})$. Furthermore, if the test configuration is non-trivial, the corresponding foliation would not be trivial since the $\mathbb{C}^{*}$ action on $\mathcal{M}$ is not along the leaf direction given by the orthogonal complement of the tangent space of the fibers with respect to $\Omega+\sqrt{-1} \partial \bar{\partial} \Phi$. Thus, in this case, we do get a non-trivial geodesic ray. Since $\Phi$ is smooth on $\mathcal{M}$, the geodesic ray is parallel to the algebraic ray defined simply by pulling back $\Omega$ through the $\mathbb{C}^{*}$ action. Thus the ray is tamed by the test configuration.

Proof of Theorem 6. First it is easy to see that the above arguments still go through if we change the general framework replacing $C^{\bar{\epsilon}}$ by $C^{k}$ for $k$ large. First of all, following the proof of $[\mathbf{8}]$, the one-to-one correspondence before Lemma 7 becomes that a $C^{k+1}$ solution $\Phi$ corresponds to a map $G$ which is only $C^{k}$ along $M$ (i.e., the corresponding exact LS-graph is only of class $C^{k}$ ). Now we consider $C^{k}$ neighborhood $\mathcal{N}^{k}\left(R_{0}\right)$ of a $C^{k}$ totally real submanifold $R_{0}$. This could be identified with an open set of the space $\Gamma^{k}\left(N R_{0}\right)$ of all $C^{k}$ sections of $N R_{0}$ (in our case, $R_{0}$ will be the exact LS-graphs defined over $\partial D$ corresponding to the boundary value of a $C^{k+1}$ solution to the homogeneous Monge-Ampère equation). More precisely, define

$$
\Gamma_{r}^{k}\left(N R_{0}\right)=\left\{X \in \Gamma^{k}\left(N R_{0}\right) \mid\|X\|_{C^{k}}<r\right\} .
$$

We also choose $r$ sufficiently small, and define $\mathcal{N}^{k}\left(R_{0}\right)$ to be the image of $\Gamma_{r}^{k}\left(N R_{0}\right)$ under the above identification. Define $\mathcal{F}_{s}, \mathcal{B}$ and $\mathcal{J}$ the same as before, where $s$ should be no bigger than $k$. The previous section $s(u, J, R)=\left(\bar{\partial}_{J} u,\left.\phi_{R}^{-1} \circ u\right|_{\partial D}\right)$ is $C^{k}$ in $u$, and $C^{\infty}$ in the remaining variables. The submanifold of $(\partial D, E)_{s-\frac{1}{2}}$

$$
\left(\partial D, R_{0}\right)_{s-\frac{1}{2}}:=(\partial D, E)_{s-\frac{1}{2}} \cap C^{0}\left(\partial D, R_{0}\right)
$$


is a $C^{k}$ Banach manifold. So the implicit function theorem implies that $s^{-1}\left(\{0\} \times\left(\partial D, R_{0}\right)_{s-\frac{1}{2}}\right)$ is $C^{k}$ Banach manifold near $\left(u_{0}, J_{0}, R_{0}\right)$. Then the projection map $\pi_{2}: s^{-1}\left(\{0\} \times\left(\partial D, R_{0}\right)_{s-\frac{1}{2}}\right) \rightarrow \mathcal{J} \times \mathcal{N}^{k}\left(R_{0}\right)$ is a $C^{k}$ Fredholm map of index $2 n . \pi_{2}^{-1}\left(J_{0}, R_{0}\right)$ is a $C^{k}$ manifold near $\left(u_{0}, J_{0}, R_{0}\right)$ since it is parametrized by $M$ under the map $G$. Since these consists of regular holomorphic discs, we can find a neighborhood $\mathcal{V}$ of $\left(J_{0}, R_{0}\right)$, such that there exists a $C^{k}$ diffeomorphism $F: \mathcal{V} \times M \rightarrow \pi_{2}^{-1}(\mathcal{V})$. Furthermore, since $s$ is smooth in $\mathcal{J}$ and $\mathcal{N}^{k}\left(R_{0}\right)$ direction, $F$ could be chosen to depend smoothly on $\mathcal{V}$-variable. By using once again the correspondence before Lemma 7 , we arrive at that $C^{k+1}$ perturbation of the boundary condition gives rise to a $C^{k+1}$ perturbation of the solution to (A), and the dependence is smooth.

With this at hand, we can define a smooth map $S$ from the open set of elements $(r, \phi)$ in $[0,1] \times C^{k+1}(M ; \mathbb{R})$ such that $\phi$ is the boundary value of a $C^{k+1}$ solution of the homogeneous Monge-Ampère equation for $\mathcal{M}(r)$ to $\tilde{\mathcal{H}}_{0}$. Given $r$ and $\phi \in C^{k+1}(M ; \mathbb{R})$ in this open set, we take the solution $\Phi$, and then restrict to the central fiber to obtain an element in $\tilde{\mathcal{H}}_{0}$. For the product test configuration $\left(M_{0}, J\left|M_{0}, \Omega\right| M_{0}\right) \times D$ with the $\mathbb{C}^{*}$ action on $M_{0}$, any $\phi \in \tilde{\mathcal{H}}_{0}$ forms a trivial solution, and $S(\phi)=\phi$. The derivative with respect to the second variable $\left(D_{2} S\right)_{(0, \phi)}: C^{k+1}(M ; \mathbb{R}) \rightarrow \tilde{\mathcal{H}}_{0}$ is then clearly surjective, with an obvious right inverse which is the inclusion map. By the implicit function theorem together with the previous paragraph, we conclude that for any $\phi \in \tilde{\mathcal{H}}_{0}$, there exists a neighborhood $\mathcal{U}$ of $\phi$, an $r>0$, and a smooth map $T: \mathcal{U} \rightarrow C^{k+1}(\mathcal{M}(r) ; \mathbb{R})$, such that the image of $T$ consists of solutions to (A) on $\mathcal{M}(r)$.

Remark 8. An interesting question is: Given a sequence of Kähler potentials in $\mathcal{H}$ which is bounded in the sense of Cheeger-Gromov, but not bounded in the holomorphic category. Does there exist a point in the "sphere at infinity" which reflects this non-compactness or degeneracy?

\section{References}

[1] C. Arezzo and G. Tian, Infinite geodesic rays in the space of Kähler potentials, Ann. Sc. Norm. Super. Pisa Cl. Sci. (5) 2(4) (2003), 617-630.

[2] E. Calabi and X.-X. Chen, Space of Kähler metrics and Calabi flow, J. Diff. Geom. 61(2) (2002), 173-193.

[3] X.-X. Chen, The space of Kähler metrics, J. Diff. Geom. 56(2) (2000), 189-234.

[4] X.-X. Chen, Space of Kähler metrics III - on the lower bound of the Calabi energy and geodesic distance, arxiv:math/0606228.

[5] X.-X. Chen and Y.-D. Tang, Test configurations and geodesic rays, arxiv:math/ 0707.4149

[6] X.-X. Chen and G. Tian, Geometry of Kähler metrics and foliations by holomorphic discs, arxiv:math/0409433. 
[7] S. K. Donaldson, Symmetric spaces, Kähler geometry and Hamiltonian dynamics, Northern California Symplectic Geometry Seminar, 13-33, Amer. Math. Soc. Transl. Ser. 2, 196, American Mathematical Society, Providence, RI, 1999.

[8] S. K. Donaldson, Holomorphic discs and the complex Monge-Ampre equation, J. Symplectic Geom. 1(2) (2002), 171-196.

[9] S. K. Donaldson, Scalar curvature and stability of toric varieties, J. Diff. Geom. 62(2) (2002), 289-349.

[10] S. K. Donaldson, Scalar curvature and projective embeddings, II, Q. J. Math. 56(3) (2005), 345-356.

[11] T. Mabuchi, Some symplectic geometry on compact Kähler manifolds I. Osaka, J. Math. 24(2), (1987), 227-252.

[12] T. Mabuchi, An energy-theoretic approach to the Hitchin-Kobayashi correspondence for manifolds, I, Invent. Math. 159(2) (2005), 225-243.

[13] Yong-Geun Oh, Fredholm theory of holomorphic discs under the perturbation of boundary conditions, Math. Z. 222(3) (1996), 505-520.

[14] D. H. Phong and J. Sturm, Test configurations for K-stability and geodesic rays, arxiv:math/0606423.

[15] D. H. Phong and J. Sturm, On the regularity of geodesic rays associated to test configurations, arxiv:math/07073956.

[16] S. Semmes, Complex Monge-Ampère equations and sympletic manifolds, Amer. J. Math. 114(3), (1992), 495-550.

[17] J. Song and S. Zelditch, Test configurations, large deviations and geodesic rays on toric varieties, arxiv:math/0712.3599.

[18] G. Tian, Kähler-Einstein metrics with positive scalar curvature, Invent. Math. 130 (1997), 1-39.

Department of Mathematics

UNIVERSITY OF WiSCONSIN-MADISON

480 Lincoln Dr, Madison, WI 53706

E-mail address: ssun@math.wisc.edu

Received 9/8/2008, accepted 8/29/2009

The author wishes to thank his advisor Professor X.-X. Chen for suggesting this problem and for kindly turning down his request of co-authorship. He is also grateful to the referee for helpful comments on the original version of this article. 
